\title{
DESIGN OF A SIMPLE REMOTE ONLINE VOTING SYSTEM WITH TOKEN GENERATION
}

\author{
O.B. Asiamah, A. A. Adjei, Prince Yeboah and K. Singh \\ School of Physical Sciences \\ Kessben University College, Kuntanase, Ashanti, Ghana
}

\begin{abstract}
Online voting is as an election system that utilizes the internet to ensure access to a domain or website and allows the eligible voters to cast their secure and secret ballot electronically. It is done by casting a ballot through a digital system over the internet. An attempt has been made to develop an electronic voting system with token generation for authenticating the voters during elections. The voting system has the client side and the administrative side. The client side is for the voters and the administrative side is handled by election officers. The registration and verification of voters are done by the election officers by entering the required data. After these entries, the system accepts the inputs and automatically generates the token. During the election the voter's login with their generated token as the voters' ID and a password assigned to each voter. After filling the portal, the voter gets access to vote for their preferred candidate. The system does not allow multiple votes with the same generated token. As voting proceeds, the system counts the number of votes for a candidate and at the same time the total number of voters. PHP (Hypertext preprocessor) was used for implementing the online model of the application, CSS (Cascadian stylesheet) was used to beautify the pages and MySQL was used as the database engine.
\end{abstract}

Keywords: Remote Online, Voting System, Token Generation

Cite this Article: O.B. Asiamah, A. A. Adjei, Prince Yeboah and K. Singh, Design of a Simple Remote Online Voting System with Token Generation, International Journal of Advanced Research in Engineering and Technology (IJARET), 10 (6), 2019, pp 96-103.

http://iaeme.com/Home/issue/IJARET?Volume $=10 \&$ Issue $=6$

\section{INTRODUCTION}

Voting is a formal manifestation of a choice between two or more candidates or courses of action, mostly expressed through a ballot or a show one's presence [1][2]. Electronic voting is an election system that allows an eligible voter to record his or her secure and secret ballot electronically and it is done by casting a ballot through a digital system instead of ballot paper. The electronic voting system was introduced in the year 1986 in the presidency of Justice Néri da Silveira. In 1994, the computerized system of counting and totalization of the general election was being done by a central computer in the superior electoral court [3]. 
Under the Presidency of Justice Marco Aurélio Mendes de Farias Mello, in 1996 the first electronic voting started in Brazil [3]. The electronic voting system is now being used by more than thirty different countries in the world.

\subsection{Components of E-Voting Systems}

The components of an electronic voting system are:

- DRE - Direct Recording Electronic voting machine - is a device to record the voter's choices. The DRE is usually a touch-screen device where the voter casts his/her vote [4].

- VVPAT - Voter-Verified Paper Audit Trail - is a paper-based record of the choices selected by the voter. The VVPAT printer is hooked to the DRE and the paper record is viewable by the voter, but it is under a transparent cover so that it cannot be modified other than through the normal voting process [4].

- EMS - Election Management System - is the system responsible for the initialization of the components that collect the votes and also for the final tallying of the votes. The EMS is usually located at central point of election.

- Optical Scanner - is an optical reader that counts votes cast on paper ballots. There is usually one scanner at each polling site and one at election central (e.g., for the counting of absentee ballots1).

- DTD - Data Transport Device - is a storage device to transfer data between different components of the systems. These devices are used to transport ballot information to the

- DREs and optical scanners at the polling site and to transport voting results to the EMS. [4]

\subsection{Types of Electronic Voting Systems}

There are two types of electronic voting.

Remote online or internet voting: This is a type of electronic voting system where voting is done through the internet. This allows voters to cast ballots from anywhere in the world as long as they are connected to the internet. This offers immense convenience but exposes the system to security risks on the internet. This is because the voting environment is not controlled. It is also possible that issues of intimidation and voter impersonation arise. [5][8]

Offline EVM voting: This type of electronic voting is done using Electronic Voting Machines (EVM) at polling booths [7].

\section{METHODOLOGY}

The methodology of choice is Rapid Application Development (RAD); this is because the objectives of RAD include high speed, high quality, and lower cost. RAD highlights the use of special methods and computer tools to speed up analysis, design and implementation phases [4] [5]. Tools include Computer Assisted Software Engineering (CASE) tools, Joint Application Design (JAD) and fourth generation programming languages. All of which are inline and essential to the proposed system.

The algorithm used in the token code generation is the shuffling method. The shuffling algorithm uses a built in function in PHP called shuffle () and is used to randomize the order of the element in a set array [9]. This algorithm made the function assigns a new generated keys for the elements in the array, its removes any existing keys rather than just reordering the keys and just assigns numeric keys starting from zero to nine (0-9) and mix with characters starting from lowercase to uppercase (a-z, A-Z). It is important to have standard notations for 
modelling, documenting, and communicating decisions. Looking at the functionality of the proposed voting system with token authentication system - use case models, data flow diagrams, entity - relationship models and the context diagram have been used to specify the functionality and non-functionality of the system in this work.

\subsection{Data Flow Diagrams (DFDs)}

This display the graphical interface of the data through an information system, the relationship among the flow of data and how data is stored in particular order. Data Flow Diagram focuses on the movement of data between processes that are called Process Models.

\subsection{Context Data Flow Diagram}

This diagram is a blueprint of the main work, process and data flow of the system being modelled and it doesn't show the data store.

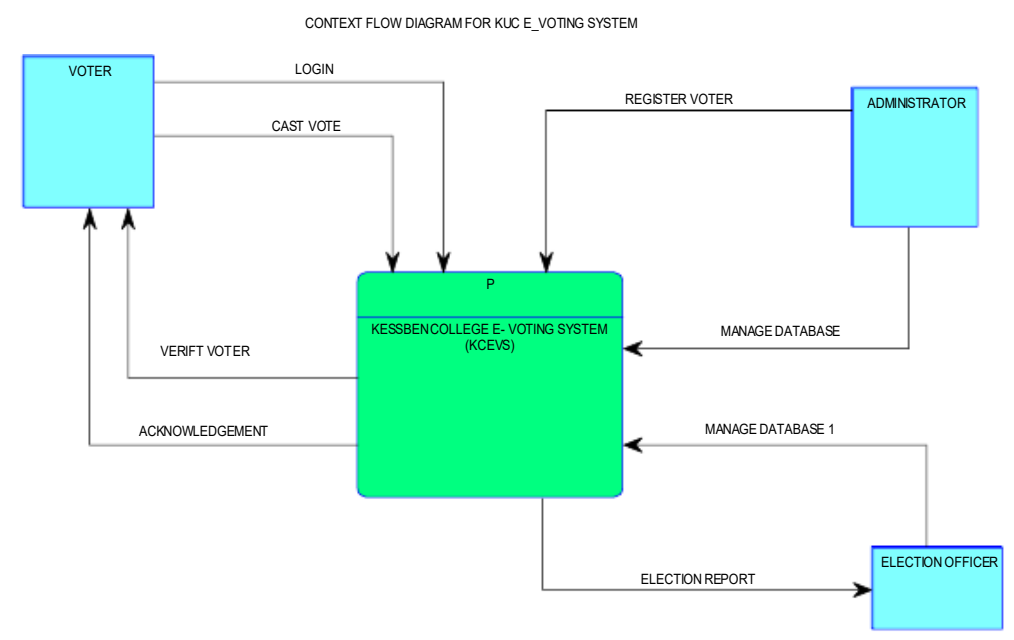

Figure 1 Context Flow Diagram For Kuc E_Voting System

Level 1 Data Flow Diagram for the Voter Enrolment Process

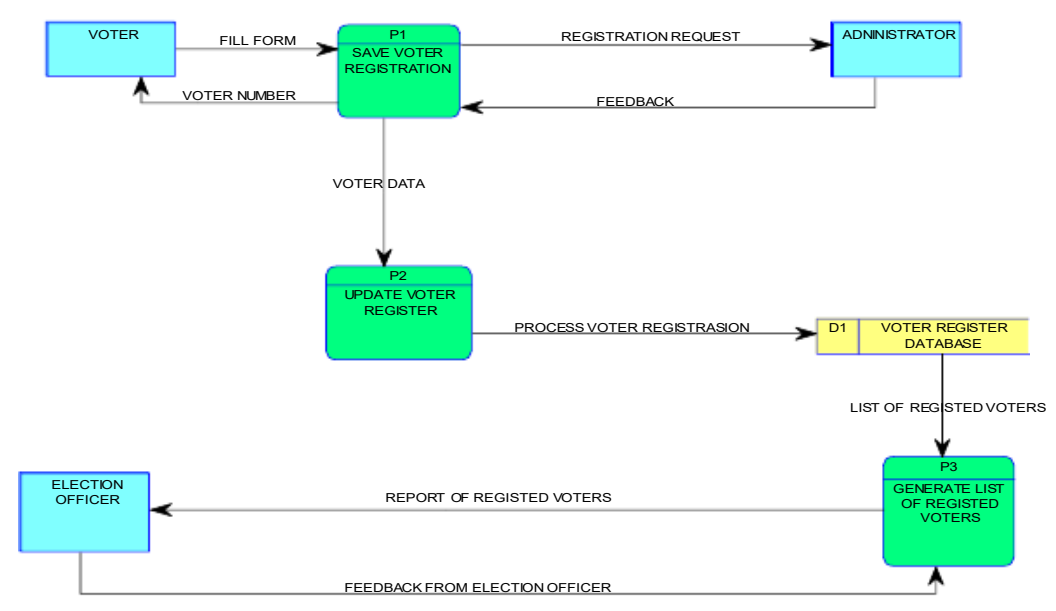

Figure 2 Level 1DFD Diagram for the Voter Enrolment Process

\subsection{Use Case Diagram}

This is a graphical work that is used to interpret the main elements and processes that make up a system. The key elements are known as "actors" and the processes are called "usecases." It 
shows which actors interact with each use case. Fig. 3 contains the breakdown of the Use Cases showing the Actors, the Input and the Output respectively:

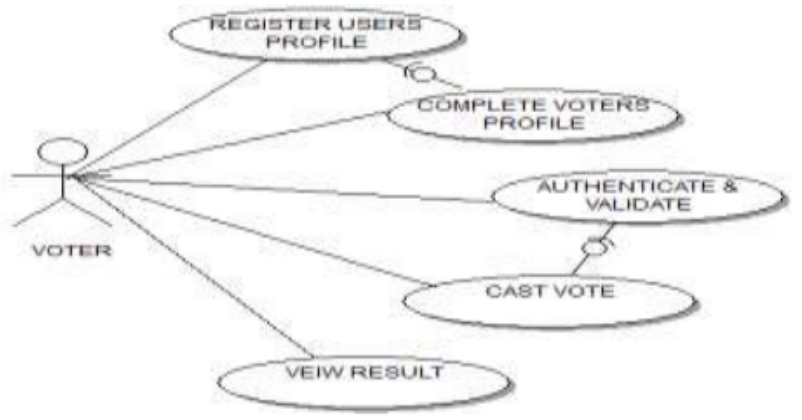

Figure 3 Use Case Diagram

\subsubsection{ACTOR: VOTER}

\subsubsection{USE CASES:}

Register Profile: Is a major functional requirement of the system. In this case, the

Voter's details and the tokens are captured on the system and stored in the database.

Complete Profile: The voter whose fingerprint template has not been captured as a result of pre-loading his/her data, it is needed to capture only his or her fingerprint template, in order to have a complete voter profile and gain a voter identification number.

Authenticate and Validate: It is a functional requirement of the system that contains the capturing of the voter's identification number and token for verification against the template that has been stored already on the database during the registration enrolment process. It compares the fingerprint templates, detects the minutiae points and matches them. When all are set and successful, the voter's id is stored in the list of authentic voters for that particular election.

Cast Vote: The voter is able to exercise their vote and choose their loved and preferred candidate for the election.

View Result: Now the voter can view results for all the elections that have been closed.

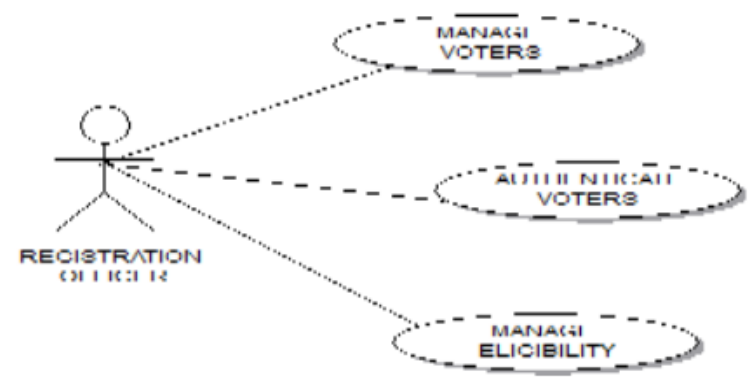

Figure 4 Use Case Diagram For Registration Officer

\subsubsection{ACTOR: REGISTRATION OFFICER}

The registration officer manages the operation of the Offline module of the Kessben University SRC Voting System.

\subsubsection{THE USE CASES:}

Manage Voters: The main function is capturing the voters' photograph and also generating secret token which is stored on the file system and the database. 
Authenticate Voters: The registration officer is responsible for verifying the eligibility of voters as they enter their unique identification number and carry out their generated token for verification to determine the real identity of the voter. After all these exercises and the voter qualifies, then the voter is authentic.

Manage Eligibility: The registration officer is authorized to create, edit, and delete eligibility criteria for an election that is created on the system.

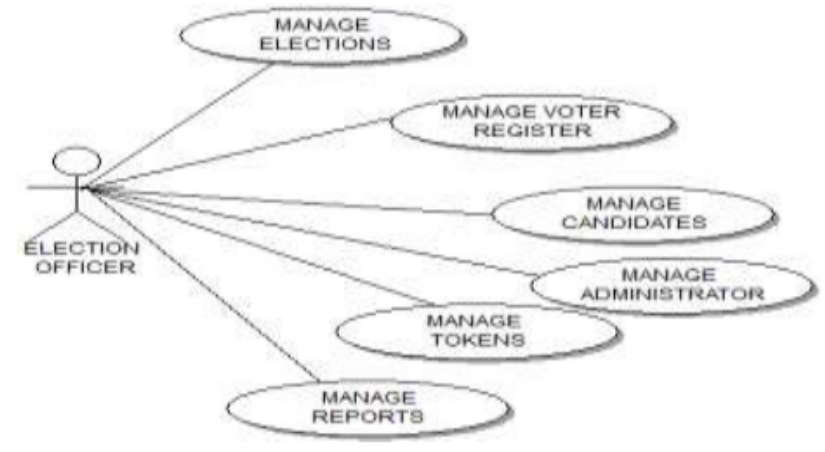

Figure 5: Use Case Diagram For Election Officer

\subsubsection{ACTOR: ELECTION OFFICER}

The Election officer is an administrator who manages the operation of the Voting

\subsubsection{USE CASES:}

Manage Elections: The election officers supposed to create a new election and manage their lifecycle. The sub-function of creating elections on the system is the operation to create ballots or offices or posts for which candidates would be able to contest for.

Manage Voter Register: There is the voter register which is the output of any voting System desktop module connected to the database. Which is all authenticated and qualified voters' detail are automatically entered into the database or the system.

Manage Candidates: This permits the election officer to create candidates for any upcoming election which has been registered on the voting module.

Manage Administrators: This function allows new administrators to be created on the system.

Manage Tokens: After successful uploading of the voter register containing the list of authenticated voters, the election officer is supposed to send out tokens which were automatically generated during the voter's register upload to all voters on the register using the mailing address or through SMS. It involves a functionality in which the individual voter's token can be retrieved and sent to the voter.

Manage Results: Managing result involve the printing of the completed election results and can also be saved as a pdf file.

\subsection{FLOW CHART}

This is a diagram that represents an algorithm, workflow or process. The flowchart shows the steps as boxes of various kinds with arrows. These charts are used in analysing, designing, documenting or managing a process or program in various fields. 


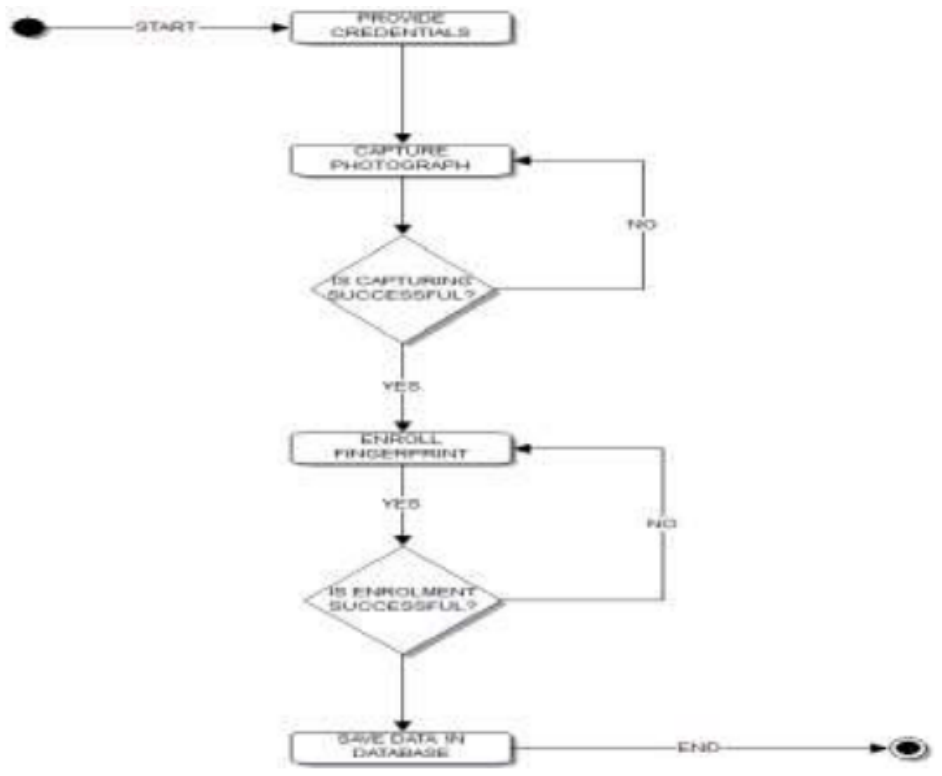

Figure 6 Flowchart for E-voting System

\section{SYSTEM ARCHITECTURE}

The system architecture is a conceptual model that defines the structure, behaviour and more view of a system. Whereas an architecture description is a formal description and representation of a system organized in a way that supports reasoning about the structure and behaviour of the system.

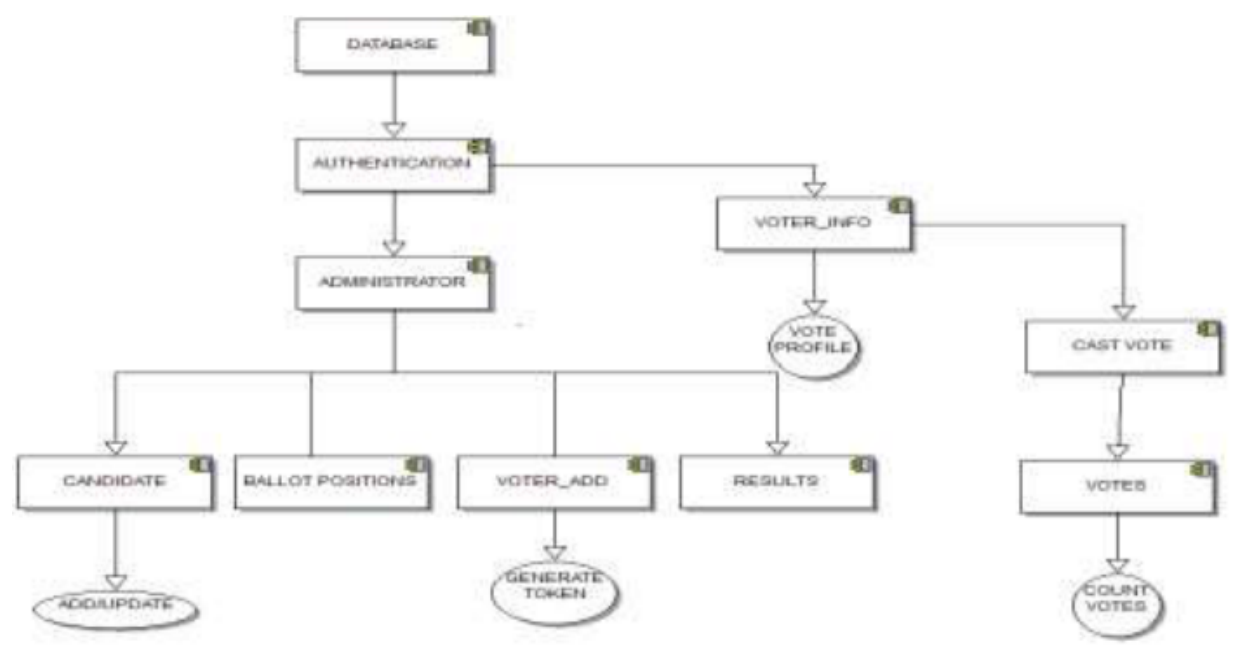

Figure 7 System Architecture Diagram for E-Voting System

\section{RESULTS AND DISCUSSION}

PHP (Hypertext pre-processor) was used for implementing the online model of the application. PHP is an object-oriented programming language. Thus, it has various libraries that helped in the designing of the user interface and also has the ability to connect with MySQL database with ease. It also helped in the security aspect by employing MD5 function for data encryption algorithms to help secure the system. JavaScript Frameworks/JavaScript

CSS (Cascadian stylesheet) was also used to beautify the pages and made them look colourful and much more attractive. 
MySQL was used as the database engine for the electronic voting system for Kessben University College (KUCEVS) project. It can be gotten for free online. MySQL database software has been highly recommended over a lot of database software since its creation.

\subsection{System Requirements}

The system requirements under which this application will work to its maximum capacity are categorized into the Hardware requirements and the Software requirements.

\subsection{Hardware Requirements}

The hardware requirements of the software include the following:

A router

A compatible computer being it a laptop or a desktop. With a minimum specification of as follows;

- GB RAM

- Dual Core processor

- 250 GB RAM

\subsection{Software Requirements}

The software requirement of the software is as followers;

a. Windows $7 / 8 / 8.1 / 10$ 64-bit operating system

- MySQL Server

- A PHP IDE

- A browser

\subsection{Personnel Training}

It does not require any special abilities to be the administrator of this election system, one needs to understand the system and how it works which is just simple and easy.

\subsubsection{Development Tools}

- XAMPP Server

- PHP STORM

- $\quad$ SUBLIME TEXT 3

\section{CONCLUSION}

This project designed and implement an electronic voting system using token or code generation that has been used in authentication on voters in an election period in Kessben University as a case study. This has provided an accurate and efficient result to Kessben University SRC election. The registration and verification of voters were done by the appointed election officers. Student's registration were done by collecting their data; names, mobile number, index number, course of study, email and the School of the University they belong to. After these entries the system used these inputs to generate a code automatically and the generated token was sent to the voter's emails and their phone's inbox as SMS. On the day election, the students logged into the system by filling his/her portal with the name and the token given as the password to get access to vote for their preferred candidate. The system 
didn't not allow multiple voting with the same token. As voting proceeded, the system counted the number of votes for a candidate and at the same time the total number of voters and after voting the system displayed the result.

\section{ACKNOWLEDGEMENTS}

Authors would like to thank the Kessben University College for providing all the necessary assistance for the completion of this work and also permission to test the system for Students Representative (SRC) election 2019.

\section{REFERENCES}

[1] Loretta Lynch (17th APRIL 2018)

[2] Kashif H.M., Dileep Kumar and Syed Muhammad Usman, "Next Generation A Secure EVoting System Based on Biometric Fingerprint Method" 2011 International Conference on Information and Intelligent Computing IPCSIT vol.18 (2011) pp .26-27

[3] Davide Balzarotti, Greg Banks, Marco Cova, Viktoria Felmetsger, Richard Kemmerer, William Robertson, Fredrik Valeur, and Giovanni Vigna "An Experience in Testing the Security of Real-world Electronic Voting Systems" pp. 5, 2010

[4] O.M. Olaniyan, T. Mapayi \& S.A. Adejumo - "A Proposed Multiple Scan Biometric Based System for Electronic Voting", African Journal Comp. \& ICT Volume 4. No. 2. Issue 1pp. 12, 2011

[5] Bolle, R., Connell, J., et al. Guide to Biometrics, Springer. 2003.

[6] F. Thompson, B. K. Alese, O. S. Adewale and O. S. Falaki - Proceedings of the International Conference on Software Engineering and Intelligent Systems 2010, July 5th-9th, Ota, Nigeria. pp. 168, 2010.

[7] F. Thompson, B. K. Alese, O. S. Adewale and O. S. Falaki - Proceedings of the International Conference on Software Engineering and Intelligent Systems 2010, July 5th-9th, Ota, Nigeria. pp. 168, 2010.

[8] M Srinivasa Rao, Venkata Siva Rao A and P Dayaker, A Case Study On Secured Embedded Voting System Using Biometric, International Journal of Civil Engineering and Technology, 8(7), 2017, pp. 313-325.

[9] Akinyemi Aminat E. - "Biometrics Based E-Voting System",Computer Science Department Final Year Project, University of Ibadan, 2014

[10] Ashraf Darwish,AliaaYoussif, MagidAlgendy,and Eman Mohamed, A Secure and Robust Prototype For Electronic Voting System, International Journal of Computer Engineering and Technology (IJCET), Volume 3, Issue 3, October - December (2012), pp. 510-519

[11] Hugh E. Williams, David Lane. - Web Database Applications with PHP and MySQL: Building Effective Database. pp. 109,111,380. 